Chapman University

Chapman University Digital Commons

2-9-2021

\title{
Asymmetric Load Carriage at Shoulder Height Affects Temporal Gait Characteristics Among Food Service Workers
}

Christopher Espino

Robin Faustino

Tiffany Franco

Angel Reign Galvan

Matthew Gothong

See next page for additional authors

Follow this and additional works at: https://digitalcommons.chapman.edu/pt_articles

Part of the Other Rehabilitation and Therapy Commons, and the Physical Therapy Commons 


\section{Asymmetric Load Carriage at Shoulder Height Affects Temporal Gait Characteristics Among Food Service Workers}

\section{Comments}

This is a pre-copy-editing, author-produced PDF of an article accepted for publication in the Proceedings of the Human Factors and Ergonomics Society Annual Meeting in 2020. The definitive publisherauthenticated version is available online at https://doi.org/10.1177/1071181320641259.

\section{Creative Commons License}

\section{(c) (1) (9)}

This work is licensed under a Creative Commons Attribution-Noncommercial-No Derivative Works 4.0 License.

\section{Copyright}

The authors

\section{Authors}

Christopher Espino, Robin Faustino, Tiffany Franco, Angel Reign Galvan, Matthew Gothong, Ennis Khaleq, Cody Occhino, and Rahul Soangra 


\title{
Asymmetric Load Carriage at Shoulder Height affects Temporal Gait Characteristics among Food Service Workers
}

\author{
Christopher Espino ${ }^{1}$, Robin Faustino ${ }^{1}$, Tiffany Franco ${ }^{1}$, Angel Reign Galvan ${ }^{1}$, Matthew Gothong ${ }^{1}$, Ennis \\ Khaleq $^{1}$, Cody Occhino ${ }^{1}$, Rahul Soangra ${ }^{* 1} \& 2$ \\ ${ }^{1}$ Crean College of Health and Behavioral Sciences, Chapman University, Orange CA 92866 \\ ${ }^{2}$ Fowler School of Engineering, Chapman University, Orange CA 92866
}

\begin{abstract}
Slips and falls are common injuries among restaurant and food service workers. It is estimated that food service workers have $60 \%$ higher rate of occupational injury or illness than workers in other industry. This study investigates effects of asymmetric load carriage at shoulder level on gait characteristics. Each participant walked with a service tray held at shoulder height. Each participant's gait was analyzed under 3 different load walking conditions, no load walking (NLW), intermediate load walking (ILW) $(2.5 \% \mathrm{BW}$ ), and maximum load walking (MLW) $(5 \% \mathrm{BW})$. We found that participants walked significantly slower with gait cycle time $1.125 \mathrm{~s}$ versus $1.150 \mathrm{~s}$ in MLW compared to $\mathrm{NLW}(\mathrm{p}<0.05)$. We also found that single limb stance time reduced significantly from $0.706 \mathrm{~s}$ during NLW to $0.694 \mathrm{~s}$ during MLW and swing time reduced significantly from $0.89 \mathrm{~s}$ to $0.86 \mathrm{~s}$. This study will help design interventions for falls due to load carriage among food service workers.
\end{abstract}

\section{Introduction}

There are over 5.4 million jobs available within the food and beverage serving industry(BLS, 2019) and it has been shown that there is a high prevalence of work-related musculoskeletal disorders among restaurant workers(Xu \& Cheng, 2014).

Food-service workers (FSW) are subject to asymmetric loading which is correlated to an increased prevalence of shoulder pain among this population(Hwang et al., 2017). Walking with an asymmetrical load at shoulder level has been shown to contribute to low back pain and knee complications(Ozgul et al., 2012), yet no previous study has simulated carrying with a tray to mimic food service workers. Previous studies have reported changes in gait pattern as a result of load carriage directly applied to other occupations(Crosbie, Flynn, \& Rutter, 1994). Park and coworkers demonstrated decreased single limb support time and increased step width in firefighters carrying their normal PPE as well as a hose over their shoulder(Park et al., 2018). Corroborating this Loverro and coworkers reported differences in hip and knee mechanics in military members carrying field-relevant symmetric loads(Loverro, Hasselquist, $\&$ Lewis, 2019). These articles suggest significant changes occur during gait carrying loads greater than $10 \%$ of participants' bodyweight, however, FSW carry a varying range of weight not exceeding $10 \mathrm{~kg}$ (Scherrer \& Wilder, 2008). While there is relevance from these load carriage articles, the purpose of this study was to investigate the effects relatively lighter asymmetric loads at shoulder level on gait for a direct application to FSW.

Variation in gait from normal behavior can predict potential fall risk in individuals(Kwon, Kwon, Park, \& Kim, 2018). Single limb stance comprises $40 \%$ of total gait cycle time(Nordin, 2001) and an asymmetrical load to the body elicits changes in gait stability mechanics(Worden, Beaudette, Brown, \& Vallis, 2016) suggesting changes in gait efficiency, which is dependent on minimizing center of gravity (COG) displacement from a mid-line to minimize energy costs (Hsu, Michael, \& Fisk, 2008). In order to maintain balance during gait, the body shifts from one side to the other through inertial
control(Hsu et al., 2008). The introduction of an asymmetrical load may affect the gait cycle because it may already displace the center of gravity laterally.

Postural adjustments are a response to maintain balance when the body is in an asymmetrical position (Aruin, 2006),

suggesting that the static position of the FSW carrying the tray without a significant load results in biomechanical changes in the body. This is further reinforced by Dreyfuss and coworkers who reported decreased single stance time when the dominant arm was restricted in a cast that weighed $<1 \%$ of participants' body weight(Dreyfuss, Elbaz, Mor, Segal, \& Calif, 2016). FSW are in a similar orientation when carrying food in that one upper extremity is restricted. FSW are tasked with balancing a tray while walking throughout a restaurant. Abbud and workers reported that during dual-task conditions there is an increased attentional demand during the single-limb support phase(Abbud, Li, \& DeMont, 2009). Balance is required to be maintained, even with the introduction of an asymmetrical load and, as previous evidence suggests (Aruin, 2006; Corrigan \& Li, 2014; Ozgul et al., 2012), the body will compensate to this asymmetry. Without accounting for the weight that is carried, it is suggested that the body position of raised hand to shoulder level in the FSW when unilaterally carrying a tray may affect the body mechanics and gait efficiency.

With the addition of a load to the body there is evidence of biomechanical changes occurring in the posture. As a result of heavy asymmetrical loading, $30 \%$ bodyweight, there is increased muscle activity on the non-carrying side(Corrigan \& $\mathrm{Li}, 2014)$. The purpose of this study is to investigate temporal changes in gait parameters such as stance time and gait cycle time. We hypothesize that external load added to tray carrying posture will affect stance time, and speed similar to as reported earlier(Demur \& Demura, 2010).

\section{Methods \\ Subjects}

Ten healthy subjects ( 7 males, 3 females) with no previous history of neurological or musculoskeletal disorders 
participated in this study. Their age ranged from 22 to 26 years $(24 \pm 2)$, height was $167 \pm 3 \mathrm{~cm}$, and weight was $71 \pm 12$ $\mathrm{kg}$. The protocol involved two trials for each condition by every subject. All subjects had to provide written consent as per Chapman University Institutional Review Board (CUIRB). Participants were then outfitted with 26 reflective markers placed on bony landmarks and key areas on the body.

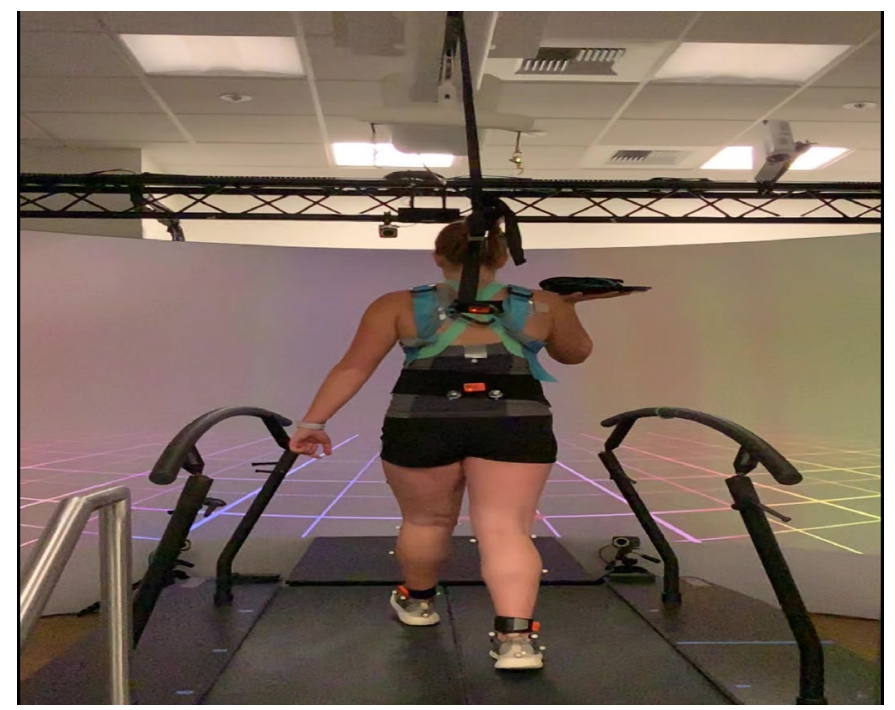

Figure 1. shows a participant carrying load on a tray

\section{Experimental Protocol}

To simulate the task of a restaurant server, each participant walked on a treadmill for one minute holding an 11-inch food service tray in three separate walking conditions: with $0 \%$ bodyweight- No Load Walking (NLW), with 2.5\% body weight- Intermediate Load Walking (ILW) and with 5\% bodyweight -Maximum Load Waling (MLW). The rationale of choosing these weights was to simulate conditions faced by food service workers. A random number generator was used to randomly assign the order of participants and the order of trials to each participant. A brief rest period was given to each participant between each of the three conditions. During each trial, participants were given verbal encouragement and cues to ensure that shoulder height of tray was maintained with shoulder abduction, elbow flexion and wrist extension.

Marker data was collected using a motion capture video recording system to track gait cycle time, single stance time and swing time. Other variables recorded included gait speed, step length, stance time, trunk sway and pelvic tilt. All dependent variables were measured and compared between each of the three conditional trials.

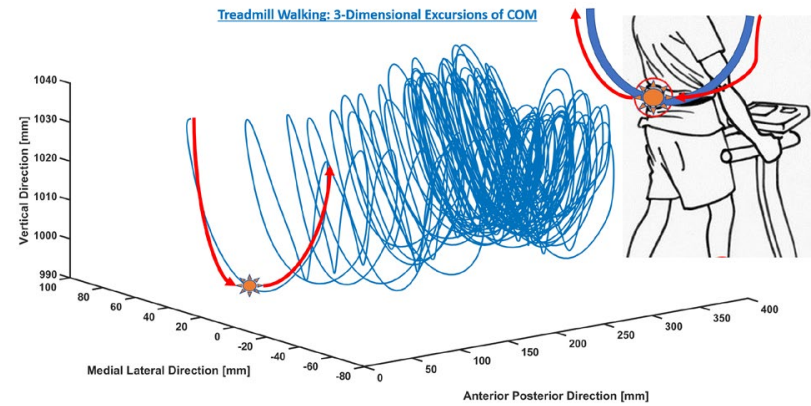

Figure 2: 3D excursions of COM during Treadmill walking

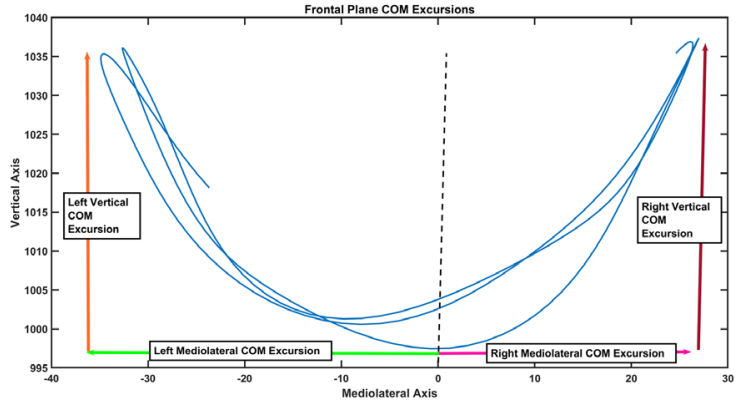

Figure 3: Frontal plane COM excursions in mediolateral and vertical directions for Right and Left side

COM was computed as mean of four pelvic markers.

Statistical procedure used was MANOVA, with alpha level set to 0.05 .

\section{Results}

During treadmill walking medial-lateral and vertical excursions of Center of Mass (COM) were computed as shown in figure 2 and figure 3 . We found the variability of COM in vertical direction reduced significantly $(\mathrm{p}=0.005)$ during load carriage conditions as shown in figure 4.

\section{COM Vertical Excursion Variability}

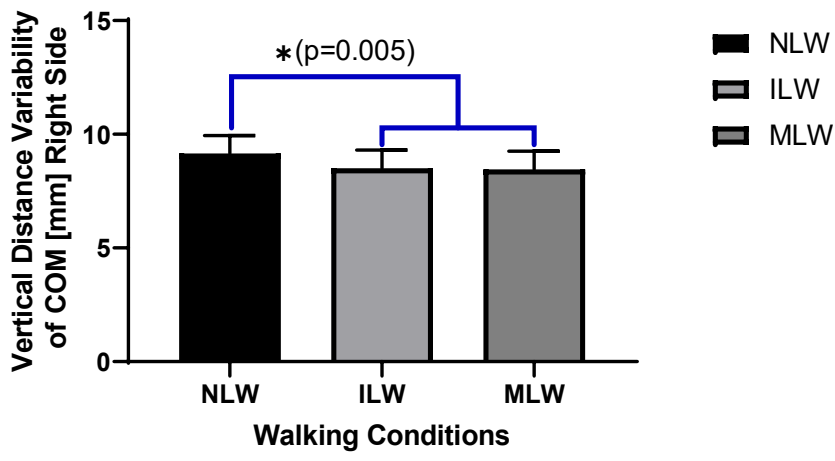

Figure 4. Shows variability in vertical excursions on the side of load carriage

We also found that Step Width variability increased significantly $(p=0.002)$ during carriage of maximum load $(5 \%$ $\mathrm{BW}$ ) as shown in Figure 5.

\section{Step Width Variability}

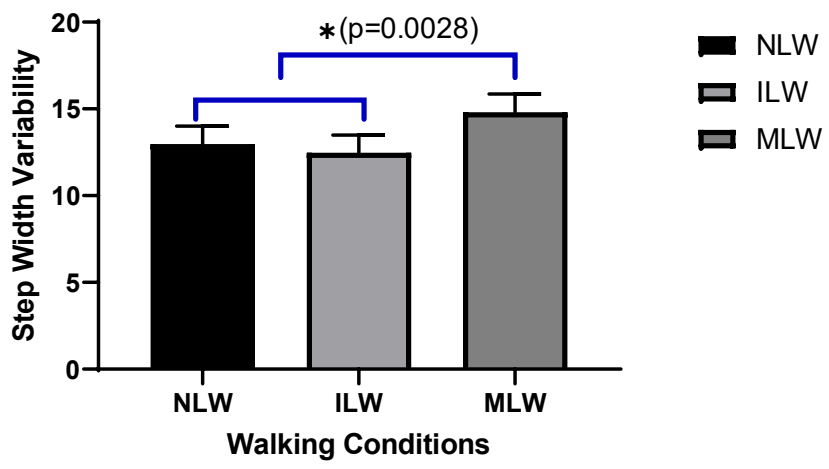


Figure 5. Step width variability increases with carrying 5\% BW on the tray

It was found that Gait cycle time reduced significantly $(p<0.05)$ for MLW condition (figure 6). We also found that Single stance time and swing time decreased significantly for MLW condition (figure 7 and 8).

\section{Gait Cycle Time}

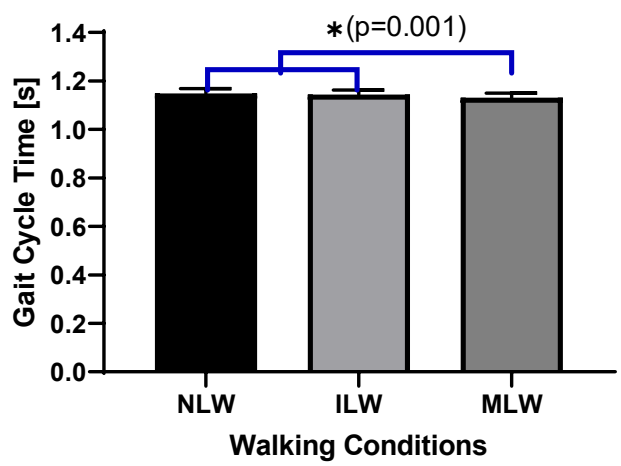

Figure 6. Gait cycle time for 3 walking conditions

\section{Single Stance Time}

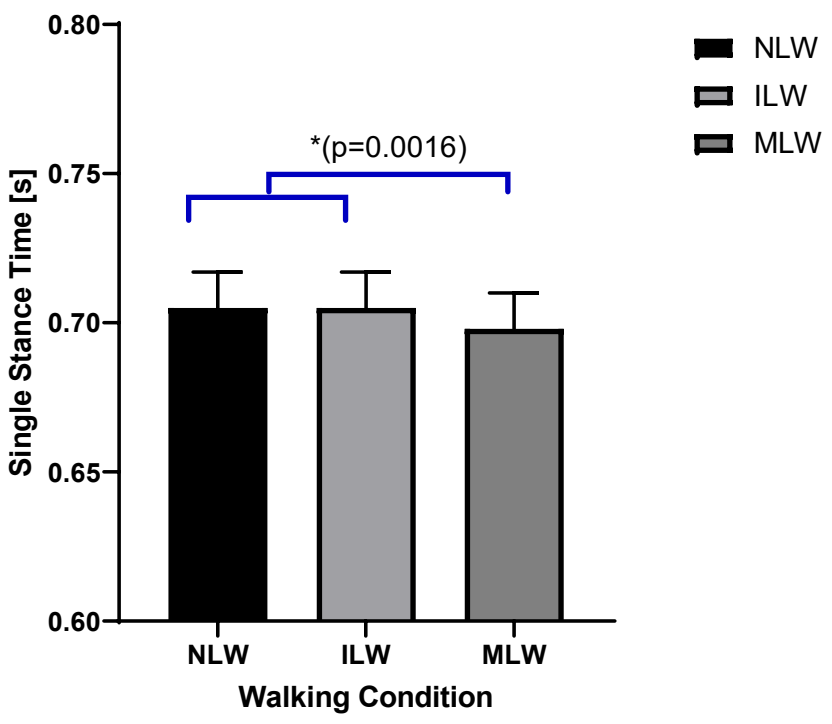

Figure 7. Single Stance time for the three walking conditions

\section{SwingTime}

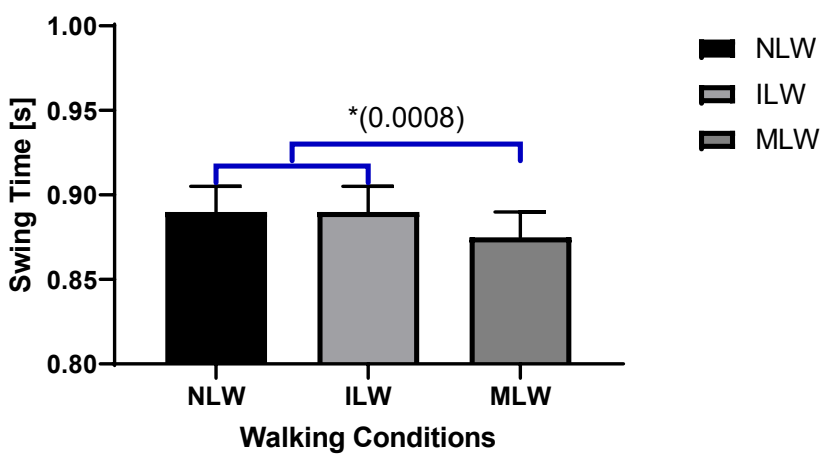

Figure 8. Swing time for three walking load carriage conditions

Discussion
The aim of this study was to investigate if carrying a tray at shoulder height influences gait. Several previous studies have investigated effects of load when carried on the trunk. There is a paucity in studies looking into the effects of a load when carried using the upper extremity in a posture resembling food service workers (Figure 1). An absence of this information potentially leaves occupational hazards unknown for FSWs.

We found that the maximum load carriage elicited significant changes in leg stance time, leg swing time, gait cycle time, step width variability and vertical excursion variability in COM. These results confirm our hypothesis that carrying load in posture similar to FSW induces alterations in gait, which may further increase risk of falls and injuries.

In this study, the weight of the load were set to $2.5 \%, 5 \%$, and a control weight of $0 \%$ of BW. Results illustrate significant changes in gait cycle time, single stance time, and swing time, variability in COM vertical excursions occur particularly with the maximal load weight of $5 \%$, as indicated earlier. Furthermore, increasing the weight of the load carried with one upper limb, overall gait mechanics may deteriorate. In addition, muscle fatigue from the ipsilateral upper and lower limb can present increased concern for injury. It has been earlier reported that individuals with altered gait patterns have up to twice the caloric expenditure for walking(Kuo \& Donelan, 2010). According to Tay and coworkers, gait changes and inefficient gait increased the $\mathrm{VO} 2$ usage in individuals carrying a load(Tay et al., 2016). It is expected that people who are subjected to multitudes of hours in the FSW position and altered gait would be subjected to less efficient movements, more fatigue and prone to higher fall risk.

This study found increased step width variability due to load carriage. Step width variability is common in individuals trying to maintain their base of support in unstable positions. Step width variability is a measure of instability and is suggested as a predictor to identify elderly who would likely fall(Maki, 1997). It is indicative of instability and compensation to maintain center of mass in the base of support. Variation in step width is also indicative of fatigue. In a study examining the effects of fatigue on gait, it was found that there was an increase in step width variance when muscles began to fatigue(Nagano, James, Sparrow, \& Begg, 2014). It may be due to muscles getting fatigued causing the body the need to mechanically widen the base of support.

The elevated load functionally fixes the limb in a position which restricts its ability to perform an arm swing. This was noticed in reduced variability in vertical excursions of COM. Arm swing play important role in counter balancing. The arm swing extension contralaterally opposes the flexion force of the leg flexion phase. The absence of arm swing can impact gait stability. The absence of upper extremity movement will reduce the efficiency of the gait cycle(Hyung, Lee, \& Kwon, 2016). For individuals in a prolonged state of limited upper extremity during gait, fatigue may subsequently increase injury.

\section{Limitations:}


Future studies may want to include experienced individuals that work in the food service industry. Individuals that are familiar with the posture and load may have altered postural strategies and gait cycles to allow a more efficient ambulation. We will further investigate normal gait and gait with suspended and fixed upper extremity to limit the confounding variable of arm impact on gait.

\section{Conclusion}

Literature regarding carrying an asymmetric load on one upper extremity is lacking. It is reported that of the \$1 spent on FSW safety $\$ 5$ are made by food service industries and restaurants. Restaurant owners can find our results helpful because it affects their employees health, health costs, and ultimately their business turnover. Clinicians can, by tailoring programs to counteract common ailments that occur in food service, help FSW efficiently resume their occupations. This study potentially helps clinicians to understand the physical stress of food service in restaurants and help make more efficient programs to rehabilitate and get them working again.

\section{References}

Abbud, G. A., Li, K. Z., \& DeMont, R. G. (2009). Attentional requirements of walking according to the gait phase and onset of auditory stimuli. Gait Posture, 30(2), 227-232. doi:10.1016/j.gaitpost.2009.05.013

Aruin, A. S. (2006). The effect of asymmetry of posture on anticipatory postural adjustments. Neurosci Lett, 401(1-2), 150-153. doi:10.1016/j.neulet.2006.03.007

BLS. (2019). Food and Beverage Serving and Related Workers : . U.S. BLS: Occupational Outlook Handbook Retrieved from https://www.bls.gov/ooh/food-preparation-andserving/food-and-beverage-serving-and-relatedworkers.htm.

Corrigan, L. P., \& Li, J. X. (2014). The effect of unilateral hockey bag carriage on the muscle activities of the trunk and lower limb of young healthy males during gait. Res Sports Med, 22(1), 23-35. doi:10.1080/15438627.2013.852094

Crosbie, J., Flynn, W., \& Rutter, L. (1994). Effect of side load carriage on the kinematics of gait. Gait \& Posture, 2(2), 103-108. doi:10.1016/0966-6362(94)90099-x

Demur, T., \& Demura, S. (2010). Relationship among gait parameters while walking with varying loads. $J$ Physiol Anthropol, 29(1), 29-34. doi:10.2114/jpa2.29.29

Dreyfuss, D., Elbaz, A., Mor, A., Segal, G., \& Calif, E. (2016). The effect of upper limb casting on gait pattern. Int J Rehabil Res, 39(2), 176-180. doi:10.1097/MRR.0000000000000155

Hsu, J. D., Michael, J., \& Fisk, J. (2008). AAOS Atlas of Orthoses and Assistive Devices E-Book: Elsevier Health Sciences.

Hwang, U. J., Kwon, O. Y., Yi, C. H., Jeon, H. S., Weon, J. H., \& Ha, S. M. (2017). Predictors of upper trapezius pain with myofascial trigger points in food service workers: The STROBE study. Medicine (Baltimore), 96(26), e7252. doi:10.1097/MD.0000000000007252

Hyung, E. J., Lee, H. O., \& Kwon, Y. J. (2016). Influence of load and carrying method on gait, specifically pelvic movement. J Phys Ther Sci, 28(7), 2059-2062. doi:10.1589/jpts.28.2059

Kuo, A. D., \& Donelan, J. M. (2010). Dynamic principles of gait and their clinical implications. Phys Ther, 90(2), 157-174. doi:10.2522/ptj.20090125

Kwon, M. S., Kwon, Y. R., Park, Y. S., \& Kim, J. W. (2018). Comparison of gait patterns in elderly fallers and non-fallers. Technol Health Care, 26(S1), 427-436. doi:10.3233/THC-174736

Loverro, K. L., Hasselquist, L., \& Lewis, C. L. (2019). Females and males use different hip and knee mechanics in response to symmetric military-relevant loads. Journal of Biomechanics, 95, 109280. doi:10.1016/j.jbiomech.2019.07.024

Maki, B. E. (1997). Gait changes in older adults: predictors of falls or indicators of fear. J Am Geriatr Soc, 45(3), 313-320. doi:10.1111/j.1532-5415.1997.tb00946.x

Nagano, H., James, L., Sparrow, W. A., \& Begg, R. K. (2014). Effects of walking-induced fatigue on gait function and tripping risks in older adults. $J$ Neuroeng Rehabil, 11, 155. doi:10.1186/1743-0003-11-155

Nordin, M. (2001). Basic biomechanics of the musculoskeletal system by Margareta Nordin Victor H. Frankel. Philadelphia: Lippincott Williams and Wililins.

Ozgul, B., Akalan, N. E., Kuchimov, S., Uygur, F., Temelli, Y., \& Polat, M. G. (2012). Effects of unilateral backpack carriage on biomechanics of gait in adolescents: a kinematic analysis. Acta Orthop Traumatol Turc, 46(4), 269-274. doi:10.3944/aott.2012.2678

Park, K., Sy, J. F., Horn, G. P., Kesler, R. M., Petrucci, M. N., Rosengren, K. S., \& Hsiao-Wecksler, E. T. (2018). Assessing gait changes in firefighters after firefighting activities and while carrying asymmetric loads. Appl Ergon, 70, 44-50. doi:10.1016/j.apergo.2018.01.016

Scherrer, M. D., \& Wilder, D. A. (2008). Training to increase safe tray carrying among cocktail servers. J Appl Behav Anal, 41(1), 131-135. doi:10.1901/jaba.2008.41-131

Tay, C. S., Lee, J. K., Teo, Y. S., Foo, P. Q., Tan, P. M., \& Kong, P. W. (2016). Using gait parameters to detect fatigue and responses to ice slurry during prolonged load carriage. Gait Posture, 43, 17-23. doi:10.1016/j.gaitpost.2015.10.010

Worden, T. A., Beaudette, S. M., Brown, S. H., \& Vallis, L. A. (2016). Estimating Gait Stability: Asymmetrical Loading Effects Measured Using Margin of Stability and Local Dynamic Stability. J Mot Behav, 48(5), 455-467. doi:10.1080/00222895.2015.1134433

Xu, Y. W., \& Cheng, A. S. (2014). An onsite ergonomics assessment for risk of work-related musculoskeletal disorders among cooks in a Chinese restaurant. Work, 48(4), 539-545. doi:10.3233/WOR-131805 